ARTICLE

\title{
Ab initio electron-two-phonon scattering in GaAs from next-to-leading order perturbation theory
}

\author{
Nien-En Lee ${ }^{1,2}$, Jin-Jian Zhou (10 ${ }^{1}$, Hsiao-Yi Chen ${ }^{1,2} \&$ Marco Bernardi ${ }^{1 凶}$
}

Electron-phonon (e-ph) interactions are usually treated in the lowest order of perturbation theory. Here we derive next-to-leading order e-ph interactions, and compute from first principles the associated electron-two-phonon (2ph) scattering rates. The derivations involve Matsubara sums of two-loop Feynman diagrams, and the numerical calculations are challenging as they involve Brillouin zone integrals over two crystal momenta and depend critically on the intermediate state lifetimes. Using Monte Carlo integration together with a self-consistent update of the intermediate state lifetimes, we compute and converge the $2 \mathrm{ph}$ scattering rates, and analyze their energy and temperature dependence. We apply our method to GaAs, a weakly polar semiconductor with dominant optical-mode long-range e-ph interactions. We find that the $2 \mathrm{ph}$ scattering rates are as large as nearly half the value of the one-phonon rates, and that including the 2 ph processes is necessary to accurately predict the electron mobility in GaAs from first principles.

\footnotetext{
${ }^{1}$ Department of Applied Physics and Materials Science, California Institute of Technology, Pasadena, CA 91125, USA. ${ }^{2}$ Department of Physics, California Institute of Technology, Pasadena, CA 91125, USA. ${ }^{凶}$ email: bmarco@caltech.edu
} 
E lectron-phonon ( $e$-ph) interactions are essential to understanding electrical transport, nonequilibrium dynamics and superconductivity. Using density functional theory (DFT) and related methods, it has become possible to compute $e-p h$ interactions from first principles, and use them to predict the carrier scattering rates and mobilities, both in simple and in complex materials with up to tens of atoms in the unit cell ${ }^{1-9}$. In the typical workflow, one takes into account only the leadingorder $e$-ph scattering processes, which involve scattering of the carriers with one phonon. Nearly all ab initio work to date has relied on such leading-order perturbation theory, tacitly neglecting higher-order $e$-ph processes.

Yet many compounds, including polar semiconductors, oxides and organic crystals, exhibit $e-p h$ interactions that cannot be treated with lowest-order perturbation theory. Polar materials are a paradigmatic case in which $e$-ph interactions due to polar optical modes-in particular, the longitudinal optical (LO) mode-are long-range $e^{10,11}$, which can lead to higher-order $e$-ph scattering and polaron formation. In weakly polar materials, such as III-V and IIVI semiconductors and high-mobility oxides, one expects that perturbation theory is still valid, but that higher-order $e$-ph processes are significant and need to be included.

Studies of second- and even third-order $e$-ph processes in semiconductors exist ${ }^{12-17}$, but they are limited to simplified models, restricted to the conduction band minimum, or only valid at zero temperature, and are therefore inadequate for quantitative predictions. In first-principles $e$-ph and charge transport calculations, next-to-leading-order effects have been typically assumed to be negligible, even though their contributions have been estimated to be important using simplified models $^{13-15,18,19}$. In materials with intermediate or strong $e-p h$ coupling, such as oxides with low room-temperature mobility and ionic compounds (e.g., alkali halides), the $e$-ph interactions can lead to regimes beyond the reach of perturbation theory, including the formation of polarons ${ }^{20}$. This coupling regime has been investigated with diagram-resummation techniques such as the cumulant method, both analytically ${ }^{21}$ and more recently ab initio $^{22-24}$.

Higher-order processes are generally important in quantum field theories of condensed matter. An example are light-matter interactions, where phonon-assisted ${ }^{25}$ and two-photon ${ }^{26,27}$ absorption have been studied extensively. Even calculations in quantum electrodynamics beyond the leading order can provide important lessons-for example, higher-order corrections are essential to accurately predict large-angle Bhabha scattering in electron-positron collisions ${ }^{28}$, and calculations up to the tenth order have been carried out to compute the magnetic moment of the electron ${ }^{29}$.

However, it is a daunting task to systematically go beyond the leading order due to the rapid increase in the number of Feynman diagrams and their computational complexity. For $e$-ph interactions, the next-to-leading order involves electron scattering events with two phonons, which requires computationally challenging Brillouin zone integrals over two crystal momenta. This computational complexity has hampered next-to-leading-order $e$-ph calculations for decades ${ }^{12}$, and the relevant next-to-leadingorder diagrams (see Fig. 1) have not yet been computed numerically from first principles.

Here we formulate and compute from first principles next-toleading-order $e-$ ph interactions, focusing on electron scattering processes involving two phonons (hereafter denoted as $2 \mathrm{ph}$ processes). We compute and analyze their contributions to the $e$-ph scattering rates, using as an ideal case study a weakly polar semiconductor, GaAs. We find that the 2 ph scattering rates are surprisingly large, and comparable in magnitude to the lowestorder rates due to one-phonon (1ph) processes. Our analysis shows that the relative importance of the $2 \mathrm{ph}$ contributions is

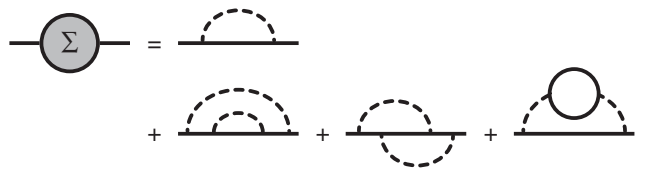

Fig. 1 Next-to-leading-order self-energy diagrams. Diagrams for the e-ph self-energy up to $\mathcal{O}\left(g^{4}\right)$, where $g$ is the e-ph coupling constant. The first two diagrams in the second row contribute to the two-phonon scattering processes.

nearly temperature independent at $200-400 \mathrm{~K}$, and rationalizes the peculiar energy dependence of the $2 \mathrm{ph}$ processes. The results are sensitive to the lifetimes of the intermediate states, which need to be included to avoid divergences due to resonance effects; we develop a self-consistent scheme to overcome this challenge. The 2 ph processes are also shown to play an important role in accurately predicting the electron mobility in GaAs. We formulate and iteratively solve a linearized Boltzmann transport equation (BTE) that includes the 2 ph processes, showing that this level of theory can correct the discrepancy with experiment of the mobility predicted with the BTE including only 1 ph processes. Our work proposes an approach for systematically improving the accuracy of ab initio $e$-ph calculations beyond the leading order. While this method is broadly applicable, it is particularly wellsuited for weakly polar (III-V and II-VI) semiconductors and high-mobility oxides, in which the weak polaron effects can be treated perturbatively.

\section{Results}

Two-phonon processes and their scattering rates. We use the Matsubara frequency sum technique ${ }^{30}$ to derive the electron selfenergy due to $e$-ph interactions up to $\mathcal{O}\left(g^{4}\right)$ (see Fig. 1), where $g$ is the $e$-ph coupling constant; our treatment focuses on computing the imaginary part of the self-energy and the related $2 \mathrm{ph}$ scattering rates. The derivations are lengthy and tedious, and are given in detail in the Supplementary Information.

All 2ph processes considered in this work consist of two consecutive $1 \mathrm{ph}$ scattering events ${ }^{12}$, as we show in Fig. 2 . They include processes in which the electron absorbs one phonon and emits another phonon, or vice versa (both processes are denoted as 1ela), and processes in which the electron emits or absorbs two phonons (denoted as $2 \mathrm{e}$ and $2 \mathrm{a}$, respectively). The intermediate electronic state accessed after the first scattering event is associated with a virtual electron that cannot be observed and whose energy can take any value. Processes are defined as on shell when the intermediate electron energy equals a band electron energy, and off shell when it does not (see Fig. 2). As we discuss below, onshell processes lead to divergent integrals, which are regularized by including the intermediate electron lifetime.

The key result of our analysis is the scattering rate due to the 2 ph processes, $\Gamma_{n \mathbf{k}}^{(2 \mathrm{ph})}$, for an electronic state with band index $n$ and crystal momentum $\mathbf{k}$, which can be expressed as

$$
\Gamma_{n \mathbf{k}}^{(2 \mathrm{ph})}=\frac{2 \pi}{\hbar} \frac{1}{N_{\Omega}^{2}} \sum_{n_{2}} \sum_{\nu \mathbf{q}} \sum_{\mu \mathbf{p}}\left[\widetilde{\Gamma}^{(1 \mathrm{el} \mathrm{a})}+\widetilde{\Gamma}^{(2 \mathrm{e})}+\widetilde{\Gamma}^{(2 \mathrm{a})}\right]
$$

where the process-resolved $2 \mathrm{ph}$ scattering rates $\widetilde{\Gamma}^{(i)}(i=1 \mathrm{e} 1 \mathrm{a}, 2 \mathrm{e}$ or $2 \mathrm{a})$ depend on the two-phonon momenta $\mathbf{q}$ and $\mathbf{p}$ and their respective mode indexes $v$ and $\mu$, and $n_{2}$ is the band index of the final electronic state, whose momentum is fixed to $\mathbf{k}^{\prime} \equiv \mathbf{k}+\mathbf{q}+\mathbf{p}$ by momentum conservation; to correctly normalize the sum, we divide it by $N_{\Omega}^{2}$, the number of points sampled in the $(\mathbf{q}, \mathbf{p})$ space. 


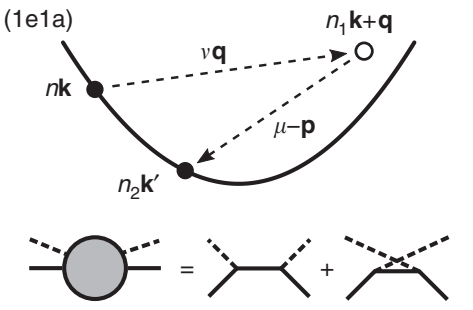

(2e)

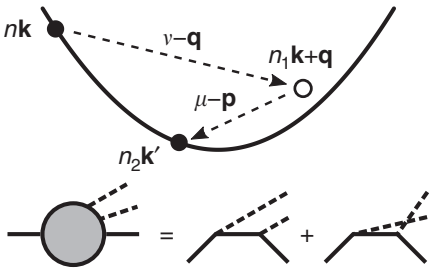

(2a)

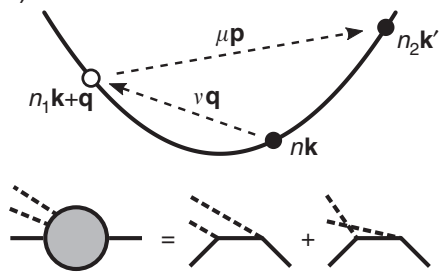

Fig. 2 Electron-two-phonon scattering processes. Two-phonon scattering processes considered in this work, including one-phonon absorption plus onephonon emission (left panel, labelled 1e1a), two-phonon emission (middle panel, labelled 2e) and two-phonon absorption (right panel, labelled 2a). Each of these three processes comprises two interfering scattering channels, only one of which is shown in the band structure schematics. The lower panels show the corresponding Feynman diagrams with two external phonon lines. Note that the intermediate state does not need to be on-shell; of the three processes shown here, only the $2 a$ is on shell.

The process-resolved $2 \mathrm{ph}$ scattering rates are defined as

$$
\widetilde{\Gamma}^{(i)}=\gamma^{(i)} \delta\left(\varepsilon_{n \mathbf{k}}-\varepsilon_{n_{2} \mathbf{k}^{\prime}}-\alpha_{\mathbf{p}}^{(i)} \omega_{\mu \mathbf{p}}-\alpha_{\mathbf{q}}^{(i)} \omega_{\nu \mathbf{q}}\right),
$$

where $\varepsilon$ are electron energies relative to the chemical potential and $\omega$ are phonon energies; the delta function imposes energy conservation and the constants $\alpha$ for each process are defined as

$$
\begin{aligned}
& \alpha_{\mathbf{p}}^{(\mathrm{lela})}=1, \quad \alpha_{\mathbf{p}}^{(2 \mathrm{e})}=1, \quad \alpha_{\mathbf{p}}^{(2 \mathrm{a})}=-1, \\
& \alpha_{\mathbf{q}}^{(1 \mathrm{ela})}=-1, \quad \alpha_{\mathbf{q}}^{(2 \mathrm{e})}=1, \quad \alpha_{\mathbf{q}}^{(2 \mathrm{a})}=-1 .
\end{aligned}
$$

The square amplitudes of the three processes are

$$
\begin{aligned}
\gamma^{(i)}= & A^{(i)} \times \\
& \mid \sum_{n_{1}}\left(\frac{g_{n_{1} n \nu}(\mathbf{k}, \mathbf{q}) g_{n_{2} n_{1} \mu}(\mathbf{k}+\mathbf{q}, \mathbf{p})}{\varepsilon_{n_{2} \mathbf{k}^{\prime}}-\varepsilon_{n_{1} \mathbf{k}+\mathbf{q}}+\alpha_{\mathbf{p}}^{(i)} \omega_{\mu \mathbf{p}}+i \eta-\Sigma_{n_{1} \mathbf{k}+\mathbf{q}}}\right. \\
& \left.+\frac{g_{n_{1} n \mu}(\mathbf{k}, \mathbf{p}) g_{n_{2} n_{1} \nu}(\mathbf{k}+\mathbf{p}, \mathbf{q})}{\varepsilon_{n_{2} \mathbf{k}^{\prime}}-\varepsilon_{n_{1} \mathbf{k}+\mathbf{p}}+\alpha_{\mathbf{q}}^{(i)} \omega_{\nu \mathbf{q}}+i \eta-\Sigma_{n_{1} \mathbf{k}+\mathbf{p}}}\right)\left.\right|^{2},
\end{aligned}
$$

where $n_{1}$ is the band index, $\Sigma$ is the self-energy of the intermediate electronic state and $\eta$ is a positive infinitesimal. The prefactors $A^{(i)}$ contain the thermal occupation numbers of electrons and phonons (denoted by $f$ and $N$, respectively) and are defined as:

$$
\begin{array}{r}
A^{(1 \mathrm{e} l \mathrm{a})}=N_{\nu \mathbf{q}}+N_{\nu \mathbf{q}} N_{\mu \mathbf{p}}+N_{\mu \mathbf{p}} f_{n_{2} \mathbf{k}^{\prime}}-N_{\nu \mathbf{q}} f_{n_{2} \mathbf{k}^{\prime}}, \\
A^{(2 \mathrm{e})}=\frac{1}{2}\left[\left(1+N_{\nu \mathbf{q}}\right)\left(1+N_{\mu \mathbf{p}}-f_{n_{2} \mathbf{k}^{\prime}}\right)-N_{\mu \mathbf{p}} f_{n_{2} \mathbf{k}^{\prime}}\right], \\
A^{(2 \mathrm{a})}=\frac{1}{2}\left[N_{\nu \mathbf{q}}\left(N_{\mu \mathbf{p}}+f_{n_{2} \mathbf{k}^{\prime}}\right)+\left(1+N_{\mu \mathbf{p}}\right) f_{n_{2} \mathbf{k}^{\prime}}\right] .
\end{array}
$$

Let us examine the process amplitudes in Eq. (3). Since all three processes have similar expressions, we focus on a 1ela process in which the electron absorbs one phonon and then emits another as an example, though our considerations are general. In this process, which is shown schematically in Fig. 3, an electron first absorbs a phonon with energy $\omega_{v \mathbf{q}}$, transitioning to an intermediate state $\left|n_{1} \mathbf{k}+\mathbf{q}\right\rangle$ with energy $E=\varepsilon_{n \mathbf{k}}+\omega_{v \mathbf{q}}$, and then emits a phonon with energy $\omega_{\mu \mathbf{p}}$, reaching the final state with energy $\varepsilon_{n_{2} \mathbf{k}^{\prime}}$. Note that the energy $E$ of the intermediate (virtual) electron does not need to match its band energy $\varepsilon_{n_{1} \mathbf{k}+\mathbf{q}}$. The amplitude for this process, from Eq. (3), reads

$$
\frac{g_{n_{1} n \nu}(\mathbf{k}, \mathbf{q}) g_{n_{2} n_{1} \mu}(\mathbf{k}+\mathbf{q}, \mathbf{p})}{\varepsilon_{n_{2} \mathbf{k}^{\prime}}+\omega_{\mu \mathbf{p}}-\varepsilon_{n_{1} \mathbf{k}+\mathbf{q}}-\Sigma_{n_{1} \mathbf{k}+\mathbf{q}}},
$$

where we omitted the in infinitesimal for clarity. This expression can be understood as follows: the two $e$-ph coupling constants are associated with each of the two consecutive $1 \mathrm{ph}$ scattering events, and the denominator is due to the propagator of the intermediate electron, which is proportional to $1 /\left(E-\varepsilon_{n_{1} \mathbf{k}+\mathbf{q}}-\Sigma_{n_{1} \mathbf{k}+\mathbf{q}}\right)$. The amplitude of the $2 \mathrm{ph}$ process is thus inversely proportional to its
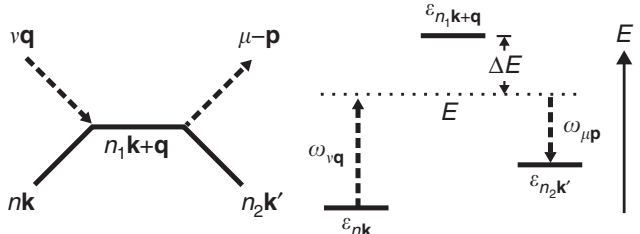

Fig. 3 Energetics of two-phonon processes. The example 1ela process discussed in the text, with its energetics shown on the right.

off-shell extent, $\Delta E=E-\varepsilon_{n_{1} \mathbf{k}+\mathbf{q}}$, implying that processes with large intermediate off-shell extents $\Delta E$ are unlikely to occur. In an on-shell $2 \mathrm{ph}$ process, $E$ is equal (or very close) to the intermediate-state band energy $\varepsilon_{n_{1} \mathbf{k}+\mathbf{q}}$, resulting in a small denominator $\Delta E \simeq 0$ and thus in a large amplitude. The inverse lifetime of the intermediate state, which is proportional to $\operatorname{Im} \Sigma_{n_{1} \mathbf{k}+\mathbf{q}}$, prevents the on-shell process amplitude from diverging.

Numerical calculations and intermediate lifetime. We implement the expressions derived above in our in-house developed code, Perturbo ${ }^{31}$, and carry out first-principles calculations on GaAs (see Methods). In our approach, we sum the 2ph scattering rates in Eq. (1) over all possible pairs of phonons, and thus include both on-shell and off-shell processes on the same footing. The treatment of the intermediate lifetimes in our implementation deserves a detailed discussion. When the intermediate state is on shell, the scattering process results in resonance effects, and as discussed above the intermediate state lifetime is crucial to prevent the 2 ph scattering rate from diverging. Here and below, the intermediate state lifetime is defined as the inverse scattering rate of the intermediate state, which is obtained from the imaginary part of its self-energy as $2 \operatorname{Im} \Sigma / \hbar$.

The 2 ph scattering rates depend on the intermediate state selfenergy, $\Sigma$ in the denominator of Eq. (3), whose value needs to be chosen carefully. We neglect its real part, which only corrects the band structure and barely affects the $2 \mathrm{ph}$ calculation. The imaginary part of $\Sigma$ includes in principle scattering effects from all possible sources. In practice, we approximate $\operatorname{Im} \Sigma$ with the total $e$-ph scattering rate, including both the lowest-order (1ph) rates and the $2 \mathrm{ph}$ rates, using $|\operatorname{Im} \Sigma|=\hbar / 2\left[\Gamma^{(1 \mathrm{ph})}+\Gamma^{(2 \mathrm{ph})}\right]$, where $\Gamma^{(1 \mathrm{ph})}$ is the usual $1 \mathrm{ph}$ scattering rate $^{3}$. This approach makes Eq. (1) a self-consistent problem. We iterate Eq. (1) until the 2ph scattering rate equals the $2 \mathrm{ph}$ contribution to the intermediate state lifetime. In each iteration $m$, the lifetime is due to the sum of the lowest order plus the $2 \mathrm{ph}$ scattering rates at the previous iteration, namely, $|\operatorname{Im} \Sigma(m)|=\hbar / 2\left[\Gamma^{(1 \mathrm{ph})}+\Gamma^{(2 \mathrm{ph})}(m-1)\right]$. The 


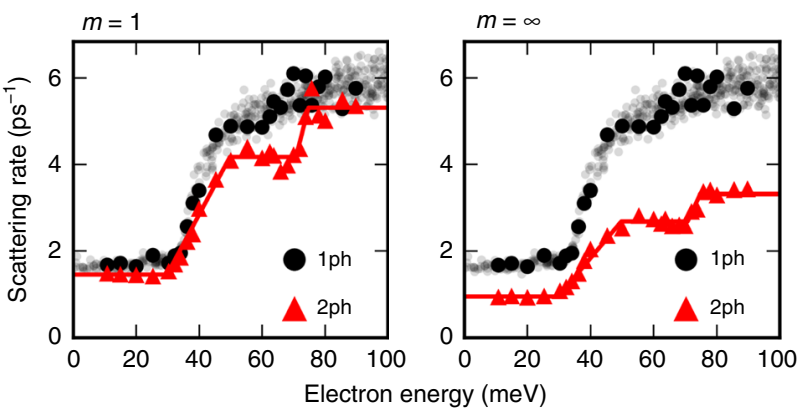

Fig. 4 Calculated two-phonon scattering rates. Calculated 2ph scattering rates, $\Gamma_{n \mathbf{k}}^{(2 \mathrm{ph})}$ in Eq. (1), for electrons in GaAs at $300 \mathrm{~K}$. The zero of the energy axis is the conduction band minimum. The left panel shows the first iteration, and the right panel the final result after converging the intermediate lifetime update procedure. The lowest-order (1ph) e-ph scattering rates are also given for comparison.

initial $\Gamma^{(2 \mathrm{ph})}$ is set to zero, and the convergence process is performed separately at each temperature.

Analysis of the two-phonon scattering rates. Figure 4 shows the first iteration and the converged result for the 2 ph scattering rates in GaAs at $300 \mathrm{~K}$, for states near the bottom of the conduction band (see Methods). In this energy range, the $e$-ph interactions in GaAs are dominated by the long-range Fröhlich interaction due to the LO mode ${ }^{3}$, with nearly negligible contributions from all other phonon modes. The converged 2 ph scattering rates are surprisingly large-they are smaller than the leading-order $1 \mathrm{ph}$ rate, thus justifying the perturbative approach, but they are nearly half the value of the $1 \mathrm{ph}$ rates at all energies. We find that the rainbow diagram (the first diagram in the second row of Fig. 1) provides the dominant contribution to the 2 ph scattering rate, while the contribution from the vertex-correction diagram (the second diagram in the second row of Fig. 1) is negligible, in agreement with Migdal's theorem. Therefore, lowest-order perturbation theory can capture only part of the dynamical processes due to the long-range $e$-ph interactions with the LO mode in GaAs. Though the results shown in Fig. 4 include only the dominant $e-$ ph interaction with the LO mode, we have verified that including all phonon modes gives nearly unchanged $2 \mathrm{ph}$ scattering rates in GaAs (see Supplementary Fig. 1).

The 2 ph scattering rate exhibits a trend as a function of energy with three plateaus near the bottom of the conduction band. This trend is a consequence of the dominant $e$-ph interactions in $\mathrm{GaAs}$, which consist of absorption or emission events of LO phonons with energy $\omega_{\mathrm{LO}} \approx 35 \mathrm{meV}$. To rationalize the energy dependence of the $2 \mathrm{ph}$ scattering rate, we define three energy regions, denoted as I, II and III in Figs. 5 and 6, which correspond respectively to electron energies below $\omega_{\mathrm{LO}}$, between $\omega_{\mathrm{LO}}$ and $2 \omega_{\mathrm{LO}}$, and greater than $2 \omega_{\mathrm{LO}}$ referenced to the conduction band minimum, which is taken hereafter to be the electron energy zero. The band structure in all three energy regions is nearly parabolic. We plot the rates of each of the $1 \mathrm{e} 1 \mathrm{a}, 2 \mathrm{e}$ and $2 \mathrm{a}$ process contributions in Fig. 5, and sketch the LO phonon emission and absorption processes in each energy region in Fig. 6 .

In region I, the electrons possess an energy smaller than $\omega_{\mathrm{LO}}$, and thus cannot emit two LO phonons since this would require a final state in the band gap; the rate of the 2e process is accordingly negligible in region I. The $2 \mathrm{a}$ process in which the electron absorbs two LO phonons is active in region $\mathrm{I}$, but it is thermally activated and weak at $300 \mathrm{~K}$ since $\omega_{\mathrm{LO}} \approx 35 \mathrm{meV}$. The $2 \mathrm{a}$ process is nearly energy independent, and weak throughout the three regions. The 1ela process in which an electron absorbs and them emits an LO phonon is the dominant contribution in region I.

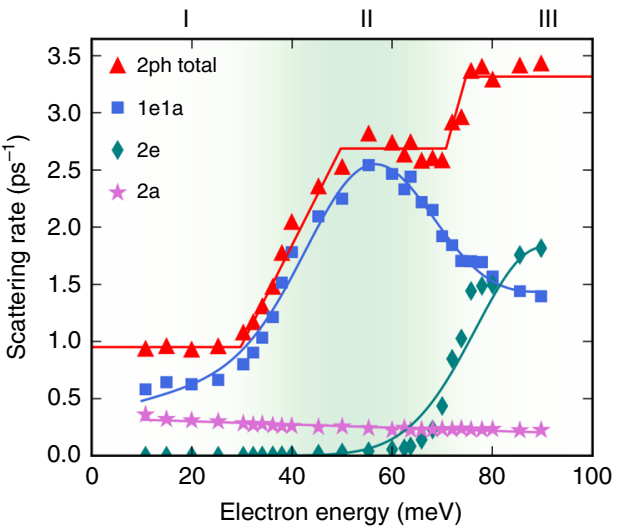

Fig. 5 Process-resolved two-phonon scattering rate. Contributions to the 2 ph scattering rate in GaAs at $300 \mathrm{~K}$. The scattering rates of the 1e1a, $2 \mathrm{e}$ and $2 \mathrm{a}$ processes are shown, together with their sum, the total $2 \mathrm{ph}$ scattering rate. The solid curves are fits of the data drawn to guide the eye.
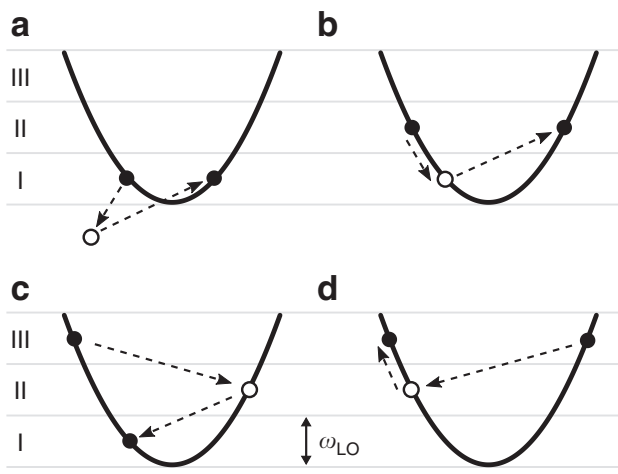

Fig. 6 Schematics of two-phonon processes. Schematics of selected 2ph processes in energy region I (a), region $\mathrm{II}(\mathbf{b})$, and region III $(\mathbf{c}, \mathbf{d})$.

Recall that the 2 ph rate is inversely proportional to the square of the off-shell extent, $\Delta E^{2}$. The 1ela channel in which an LO phonon is first emitted and then absorbed is thus suppressed in region I since its intermediate state, resulting from one LO phonon emission, is always off shell, as shown in Fig. 6a.

In region II, the 1ela scattering rate increases rapidly at energy $\omega_{\mathrm{LO}}=35 \mathrm{meV}$ because the intermediate state accessed after emitting an LO phonon can be on-shell, as shown in Fig. 6b. The rate of the $2 \mathrm{e}$ processes remains negligible in region II up to an energy of $2 \omega_{\mathrm{LO}}$. In region III, the contribution from the $2 \mathrm{e}$ process increases substantially as the electrons can emit two LO phonons and transition to the bottom of the conduction band through an on-shell process, as shown in Fig. 6c. The 1ela scattering rate drops from region II to region III due to subtle reasons related to the lifetime of the intermediate state. Recall that for on-shell processes, $\Delta E \simeq 0$, and thus the amplitude is proportional to the intermediate state lifetime (see Eq. (5)). An electron in region II can emit a phonon, transition to an on-shell intermediate state in region $\mathrm{I}$, and then absorb another phonon to transition to a final state, as shown in Fig. 6b. As the scattering rates for states in region I are considerably smaller than in the other two regions, the on-shell intermediate states have correspondingly longer lifetimes, thus giving a large amplitude to on-shell lela processes in region II. On the other hand, the 1ela processes for electrons in region III lead to intermediate states in region II or above (see Fig. 6d), which have much shorter lifetimes than in region $\mathrm{I}$, resulting in a smaller 1ela rate in region III compared to region II. These arguments allow us to rationalize the 2 ph process rates in Fig. 5. 
Let us discuss the temperature dependence of the $2 \mathrm{ph}$ scattering processes, focusing on the ratio $\Gamma^{(2 \mathrm{ph})} / \Gamma^{(1 \mathrm{ph})}$ of the $2 \mathrm{ph}$ scattering rates to the leading-order $1 \mathrm{ph}$ rates. We provide a brief analysis here and a more extensive discussion in the Supplementary Information. The temperature dependence of the $2 \mathrm{ph}$ rates originates from the intermediate state lifetimes in the denominators of Eq. (3) and the thermal factors $A^{(i)}$ in Eq. (4). The intermediate state $e$-ph scattering rate increases with temperature due to an increase in phonon number, so the intermediate state lifetimes become shorter, lowering the $2 \mathrm{ph}$ rate $\Gamma^{(2 \mathrm{ph})}$ for increasing temperatures. On the contrary, the thermal factors $A^{(i)}$, which contain factors proportional to the phonon number $N$, increase rapidly with temperature, making $\Gamma^{(2 \mathrm{ph})}$ greater at higher temperatures. In the $200-400 \mathrm{~K}$ temperature range investigated here, these two effects compensate in GaAs, resulting in nearly temperature independent $\Gamma^{(2 \mathrm{ph})} / \Gamma^{(1 \mathrm{ph})}$ ratios. We conclude that the $2 \mathrm{ph}$ processes are equally as important relative to the leading-order $1 \mathrm{ph}$ processes over a wide temperature range near room temperature.

Contribution to the electron mobility. Since the $2 \mathrm{ph}$ contributions are significant, one expects that they play a role in charge transport. Figure 7 shows the electron mobility in GaAs obtained by solving the BTE both within the relaxation time approximation (RTA) and with a more accurate iterative approach (ITA) ${ }^{4,7}$, which we have extended here to include 2ph processes (see Supplementary Information). Results are given for calculations that either neglect or include the $2 \mathrm{ph}$ contributions. The iterative solution with only $1 \mathrm{ph}$ scattering overestimates the electron mobility in GaAs by $40-80 \%$ at $200-400 \mathrm{~K}$, consistent with the results in ref. ${ }^{8}$ (and in ref. ${ }^{4}$, provided the polar correction is not included for the acoustic modes, which artificially increases the acoustic mode contribution to scattering in ref. ${ }^{4}$ and lowers the computed mobility). This result is puzzling, since the BTE can accurately predict the mobility in nonpolar semiconductors; the discrepancy with experiment is too large to be due to small errors in the electron effective mass (in our calculation, the effective mass is $0.055 m_{0}$ versus an experimental value of $0.067 m_{0}$, where $m_{0}$ is the electron mass). Redoing the mobility calculation with a band structure with the experimental effective mass of $0.067 m_{0}$ (see Supplementary Fig. 3) reduces the mobility overestimate to roughly $20 \%$ compared to experiment for the iterative BTE solution with $1 \mathrm{ph}$ scattering, and including $2 \mathrm{ph}$ processes still improves the agreement with experiment of the mobility and its temperature dependence.

This open problem is solved here by including the $2 \mathrm{ph}$ processes in the ITA (see Fig. 7), which lowers the mobility due to the additional 2ph scattering and gives mobility values in excellent agreement with experiments ${ }^{32-38}$. Both the absolute value of the mobility and its temperature dependence improve when the $2 \mathrm{ph}$ processes are included. This result implies that the agreement with experiment of the RTA with $1 \mathrm{ph}$ processes $^{3}$ is due to error compensation. We conclude that the $2 \mathrm{ph}$ contributions are crucial to improving the computed electron mobility in GaAs.

Since the long-range LO mode coupling is dominant in many polar semiconductors and oxides, we expect the 2 ph processes to be important in a wide range of polar materials. We apply our approach to $\mathrm{BaSnO}_{3}$, a weakly polar oxide with dominant LOmode $e$-ph interactions and a high room-temperature electron mobility. We compute the $2 \mathrm{ph}$ scattering rates and the mobility including 2ph processes in $\mathrm{BaSnO}_{3}$ (see Supplementary Fig. 2). Similar to GaAs, we find that the mobility computed with the iterative BTE including only $1 \mathrm{ph}$ processes is substantially higher than the experimental value, while adding 2 ph scattering significantly improves the agreement with experiment. This result confirms that our approach is broadly applicable to weakly polar semiconductors and oxides, in which perturbation theory is valid

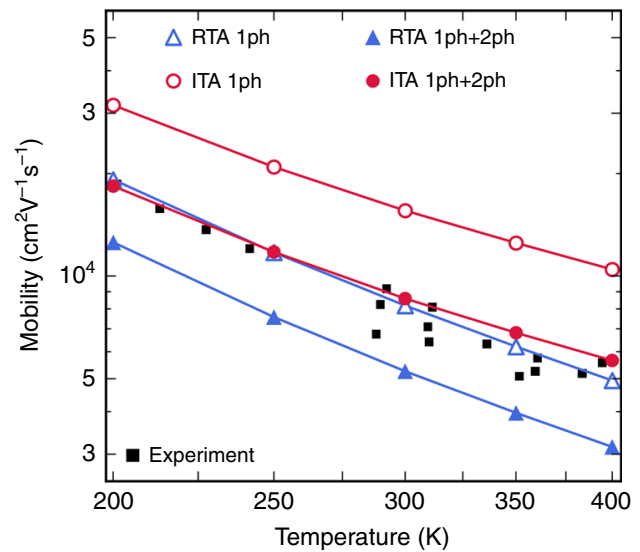

Fig. 7 Calculated electron mobility in GaAs. Electron mobility in GaAs, computed by solving the linearized BTE, both within the RTA and with an iterative approach (ITA). For each solution method, two sets of calculations are shown, one that includes only the 1ph leading-order processes and one that includes both 1ph and 2ph scattering. Experimental values are taken from refs. ${ }^{33-39}$.

and the mobility near room temperature is usually limited by scattering with the LO mode.

\section{Discussion}

Our calculations show quantitatively that the 2 ph contributions are substantial even in a weakly polar material such as GaAs in which higher-order $e$-ph interactions and polaron effects are typically neglected. The approach introduced in this work is general, and it is expected to give accurate $e$-ph scattering rates and carrier mobilities in weakly polar III-V and II-VI semiconductors and in polar oxides with high room-temperature mobility (and thus, weak polaron effects ${ }^{20}$ ); the perturbative approach is valid in these materials, where the long-range $e-p h$ interactions make next-to-leading-order effects substantial.

An open question left out for future work is whether the $2 \mathrm{ph}$ processes due to acoustic modes can be important in metals and nonpolar materials. A practical observation is that the mobility computed using only $1 \mathrm{ph}$ processes matches experiment closely in elemental metals ${ }^{2}$ and nonpolar semiconductors ${ }^{8}$; therefore, 2 ph acoustic processes are expected to be negligible, although a rigorous proof (or numerical evidence) of this point is more challenging. As discussed by Kocevar ${ }^{12}$, there are two $e$-ph perturbations contributing to $2 \mathrm{ph}$ processes. The one considered in this work is due to the first derivative of the potential with respect to lattice vibrations, taken to second order in perturbation theory, which leads to 2 ph scattering consisting of two consecutive $1 \mathrm{ph}$ scattering events. The second contribution, which is not included in this work, is due to the second derivatives of the potential with respect to lattice vibrations; it leads to a direct $2 \mathrm{ph}$ interaction associated with the so-called Debye-Waller (DW) vertex, which represents an electron interacting simultaneously with two phonons ${ }^{12}$. The DW vertex leads to additional self-energy diagrams and 2 ph processes, some of which are illustrated in Fig. 8.

Due to translational invariance, for acoustic phonons in the long-wavelength limit there is a strong cancellation between the scattering processes considered here and those due to the DW interaction $^{12,39}$. This cancellation has also been hypothesized to be valid for acoustic phonons beyond the long-wavelength limit, although this point has never been proven. This result, together with the numerical evidence that $1 \mathrm{ph}$ processes are sufficient to explain the mobility in nonpolar semiconductors and elemental metals, is suggestive of a negligible role of $2 \mathrm{ph}$ acoustic processes. Since the DW vertex cannot currently be computed ab initio for 


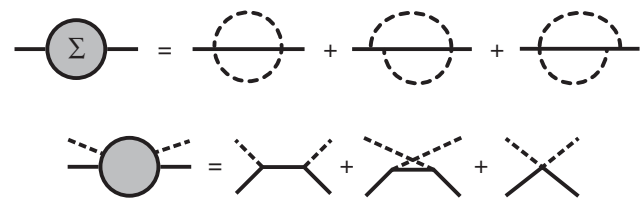

Fig. 8 Diagrams beyond this work. The top panel shows some of the additional self-energy diagrams resulting from the four-point Debye -Waller vertex, which introduces a new 1ela process interfering with the two considered in this work in the bottom panel.

two general phonon momenta, numerical studies of 2 ph processes due to the DW interaction are left out for future work. In the calculations on the two weakly polar materials considered here, $\mathrm{GaAs}$ and $\mathrm{BaSnO}_{3}$, the $\mathrm{LO}$ mode interaction is dominant, and our results are nearly unchanged if acoustic phonons are included (see Supplementary Fig. 1).

In summary, our calculations of the 2 ph scattering rates and their contribution to the mobility pave the way to studying higher-order $e-p h$ interactions and charge transport in polar materials from first principles. Together with recently proposed methods to treat charge transport in materials with polarons and stronger $e$-ph interactions ${ }^{24}$, it is clear that ab initio calculations are becoming able to investigate $e$-ph interactions and charge transport in a wide range of polar materials.

\section{Methods}

Numerical calculations. The DFT and lowest-order $e$-ph calculations in GaAs follow our previous work ${ }^{3}$. Briefly, we carry out DFT calculations in GaAs using the QUANTUM ESPRESSO code ${ }^{40}$ with a plane-wave basis set. We employ the local density approximation ${ }^{41}$ and norm-conserving pseudopotentials ${ }^{42}$. A relaxed lattice constant of $5.55 \AA$, a kinetic energy cutoff of 72 Ry and $8 \times 8 \times 8 \mathrm{k}$-point grids are used in all DFT calculations. Phonon dispersions are computed with density functional perturbation theory (DFPT) ${ }^{43}$ on an $8 \times 8 \times 8$ q-point grid. The $e$-ph coupling constants, $g_{n m v}(\mathbf{k}, \mathbf{q})$, are computed using these coarse $\mathbf{k}$ - and $\mathbf{q}$-point grids $^{3}$ using DFPT together with our in-house developed Perturbo code ${ }^{31}$, and interpolated using Wannier functions ${ }^{44}$ generated with WANNIER $90^{45}$.

To compute and converge the $2 \mathrm{ph}$ scattering rates, we use Monte Carlo integration by sampling up to 3 billion random $(\mathbf{q}, \mathbf{p})$ pairs of Brillouin zone points drawn from a Cauchy distribution ${ }^{3}$. The delta function in Eq. (2) is approximated by a Gaussian with a small broadening of $5 \mathrm{meV}$. Since in GaAs the LO phonon dominates $e$-ph scattering for electrons within $\sim 100 \mathrm{meV}$ of the conduction band edge $^{3}$, in the 2 ph scattering rate calculations we use only the long-range coupling to the LO modes ${ }^{11,46,47}$, which greatly reduces the 2 ph computational cost while giving 2 ph scattering rates nearly identical to calculations that include all phonon modes (see Supplementary Fig. 1). We use a carrier concentration of $10^{16} \mathrm{~cm}^{-3}$ in all scattering rate and mobility calculations, and set the chemical potential accordingly at each temperature. The mobility calculations are carried out by integrating over energies of up to $250 \mathrm{meV}$ above the conduction band minimum; we have verified that this energy window is sufficient to converge the mobility.

We highlight that the $2 \mathrm{ph}$ scattering rate calculations are very computationally expensive due to the large number of points sampled in the BZ integral over twophonon momenta. The average cost for computing the 2 ph scattering rate in GaAs is $\sim 500 \mathrm{CPU}$ core hours per electronic state for each iteration over the intermediate state lifetime; a typical calculation requires ten iterations to converge, for a total cost of $5000 \mathrm{CPU}$ core hours per electronic state. By contrast, it takes only 0.08 CPU core hours ( $5 \mathrm{~min}$ on a single CPU core) per electronic state to converge the $1 \mathrm{ph}$ scattering rate using our code. The computational cost of the $2 \mathrm{ph}$ scattering rates is thus approximately $10^{4}-10^{5}$ higher than the 1 ph scattering rates.

\section{Data availability}

The data that support the findings of this study are available from the corresponding author upon reasonable request.

\section{Code availability}

The code employed in this work, Perturbo, is available for download at https://perturbocode.github.io/. The 2 ph routines developed in this work are available from the corresponding author upon reasonable request.

Received: 11 April 2019; Accepted: 2 March 2020;

Published online: 30 March 2020

\section{References}

1. Bernardi, M. First-principles dynamics of electrons and phonons. Eur. Phys. J. B 89, 239 (2016).

2. Mustafa, J. I., Bernardi, M., Neaton, J. B. \& Louie, S. G. Ab initio electronic relaxation times and transport in noble metals. Phys. Rev. B 94, 155105 (2016).

3. Zhou, J.-J. \& Bernardi, M. Ab initio electron mobility and polar phonon scattering in GaAs. Phys. Rev. B 94, 201201(R) (2016).

4. Liu, T.-H., Zhou, J., Liao, B., Singh, D. J. \& Chen, G. First-principles mode-bymode analysis for electron-phonon scattering channels and mean free path spectra in GaAs. Phys. Rev. B 95, 075206 (2017).

5. Zhou, J.-J., Hellman, O. \& Bernardi, M. Electron-phonon scattering in the presence of soft modes and electron mobility in $\mathrm{SrTiO}_{3}$ perovskite from first principles. Phys. Rev. Lett. 121, 226603 (2018).

6. Lee, N.-E., Zhou, J.-J., Agapito, L. A. \& Bernardi, M. Charge transport in organic molecular semiconductors from first principles: the bandlike hole mobility in a naphthalene crystal. Phys. Rev. B 97, 115203 (2018).

7. Li, W. Electrical transport limited by electron-phonon coupling from Boltzmann transport equation: an ab initio study of $\mathrm{Si}, \mathrm{Al}$, and $\mathrm{MoS}_{2}$. Phys. Rev. B 92, 075405 (2015).

8. Ma, J., Nissimagoudar, A. S. \& Li, W. First-principles study of electron and hole mobilities of Si and GaAs. Phys. Rev. B 97, 045201 (2018).

9. Sohier, T., Campi, D., Marzari, N. \& Gibertini, M. Mobility of twodimensional materials from first principles in an accurate and automated framework. Phys. Rev. Mater. 2, 114010 (2018).

10. Fröhlich, H. Electrons in lattice fields. Adv. Phys. 3, 325-361 (1954).

11. Vogl, P. Microscopic theory of electron-phonon interaction in insulators or semiconductors. Phys. Rev. B 13, 694-704 (1976).

12. Kocevar, P. Multiphonon Scattering 167-174 (Springer US, 1980).

13. Alldredge, G. P. \& Blatt, F. J. On the role of two-phonon processes in the energy relaxation of a heated-electron distribution. Ann. Phys. 45, 191-231 (1967).

14. Sher, A. \& Thornber, K. K. Resonant electron-phonon scattering in polar semiconductors. Appl. Phys. Lett. 11, 3-5 (1967).

15. Thorbergsson, G. I. \& Sak, J. Mobility of an acoustic polaron at very low temperatures. Phys. Lett. A 116, 325-328 (1986).

16. Mora-Ramos, M. E., Rodríguez, F. J. \& Quiroga, L. Polaron properties of III-V nitride compounds: second-order effects. J. Phys. Condens. Matter 11, 8223-8238 (1999).

17. Smondyrev, M. A. Diagrams in the polaron model. Theor. Math. Phys. 68, 653-664 (1986).

18. Wang, Z. \& Mahan, G. D. Low-temperature resistivity from electron-dualphonon processes for alkali metals. Phys. Rev. B 39, 10753-10760 (1989).

19. Woods, L. M. \& Mahan, G. D. Nonlinear electron-phonon heat exchange. Phys. Rev. B 57, 7679-7685 (1998).

20. Emin, D. Polarons (Cambridge University Press, 2012).

21. Gunnarsson, O., Meden, V. \& Schönhammer, K. Corrections to Migdal's theorem for spectral functions: a cumulant treatment of the time-dependent Green's function. Phys. Rev. B 50, 10462-10473 (1994).

22. Story, S. M., Kas, J. J., Vila, F. D., Verstraete, M. J. \& Rehr, J. J. Cumulant expansion for phonon contributions to the electron spectral function. Phys. Rev. B 90, 195135 (2014).

23. Nery, J. P. et al. Quasiparticles and phonon satellites in spectral functions of semiconductors and insulators: cumulants applied to the full first-principles theory and the Fröhlich polaron. Phys. Rev. B 97, 115145 (2018).

24. Zhou, J.-J. \& Bernardi, M. Predicting charge transport in the presence of polarons: the beyond-quasiparticle regime in $\mathrm{SrTiO}_{3}$. Phys. Rev. Res. 1, 033138 (2019).

25. Noffsinger, J., Kioupakis, E., Van de Walle, C. G., Louie, S. G. \& Cohen, M. L. Phonon-assisted optical absorption in silicon from first principles. Phys. Rev. Lett. 108, 167402 (2012).

26. Murayama, M. \& Nakayama, T. Ab initio calculations of two-photon absorption spectra in semiconductors. Phys. Rev. B 52, 4986-4997 (1995).

27. Hayat, A., Ginzburg, P. \& Orenstein, M. Observation of two-photon emission from semiconductors. Nat. Photonics 2, 238 (2008).

28. Bern, Z., Dixon, L. \& Ghinculov, A. Two-loop correction to Bhabha scattering Phys. Rev. D 63, 053007 (2001).

29. Aoyama, T., Kinoshita, T. \& Nio, M. Revised and improved value of the QED tenth-order electron anomalous magnetic moment. Phys. Rev. D 97, 036001 (2018).

30. Mahan, G. D. Many-Particle Physics, 3rd edn (Springer, 2000).

31. Zhou, J.-J. et al. Perturbo: a software package for ab initio electron-phonon interactions, charge transport and ultrafast dynamics. Preprint at https://arxiv. org/abs/2002.02045 (2020).

32. Rode, D. L. Electron mobility in direct-gap polar semiconductors. Phys. Rev. B 2, 1012-1024 (1970).

33. Rode, D. L. \& Knight, S. Electron transport in GaAs. Phys. Rev. B 3, 2534-2541 (1971) 
34. Blakemore, J. S. Semiconducting and other major properties of gallium arsenide. J. Appl. Phys. 53, R123-R181 (1982).

35. Hicks, H. G. B. \& Manley, D. F. High purity GaAs by liquid phase epitaxy. Solid State Commun. 7, 1463-1465 (1969).

36. Wolfe, C. M., Stillman, G. E. \& Lindley, W. T. Electron mobility in high-purity GaAs. J. Appl. Phys. 41, 3088-3091 (1970).

37. Blood, P. Electrical properties of $n$-type epitaxial GaAs at high temperatures. Phys. Rev. B 6, 2257-2261 (1972).

38. Nichols, K. H., Yee, C. M. L. \& Wolfe, C. M. High-temperature carrier transport in n-type epitaxial GaAs. Solid-State Electron. 23, 109-116 (1980).

39. Holstein, T. Theory of ultrasonic absorption in metals: the collision-drag effect. Phys. Rev. 113, 479-496 (1959).

40. Giannozzi, P. et al. Quantum ESPRESSO: a modular and open-source software project for quantum simulations of materials. J. Phys. Condens. Matter 21, 395502 (2009).

41. Perdew, J. P. \& Zunger, A. Self-interaction correction to density-functional approximations for many-electron systems. Phys. Rev. B 23, 5048-5079 (1981).

42. Troullier, N. \& Martins, J. L. Efficient pseudopotentials for plane-wave calculations. Phys. Rev. B 43, 1993-2006 (1991).

43. Baroni, S., de Gironcoli, S., DalCorso, A. \& Giannozzi, P. Phonons and related crystal properties from density-functional perturbation theory. Rev. Mod. Phys. 73, 515-562 (2001)

44. Giustino, F., Cohen, M. L. \& Louie, S. G. Electron-phonon interaction using Wannier functions. Phys. Rev. B 76, 165108 (2007).

45. Mostofi, A. A. et al. An updated version of wannier90: A tool for obtaining maximally-localised Wannier functions. Comput. Phys. Commun. 185, 2309-2310 (2014).

46. Sjakste, J., Vast, N., Calandra, M. \& Mauri, F. Wannier interpolation of the electron-phonon matrix elements in polar semiconductors: polar-optical coupling in GaAs. Phys. Rev. B 92, 054307 (2015).

47. Verdi, C. \& Giustino, F. Fröhlich electron-phonon vertex from first principles. Phys. Rev. Lett. 115, 176401 (2015)

\section{Acknowledgements}

This work was supported by the Air Force Office of Scientific Research through the Young Investigator Program Grant FA9550-18-1-0280. J.-J.Z. and H.-Y.C. were supported by the National Science Foundation under Grant No. ACI- 1642443, which provided for code development. This research used resources of the National Energy Research Scientific Computing Center, a DOE Office of Science User Facility supported by the Office of Science of the U.S. Department of Energy under Contract No. DE-AC02$05 \mathrm{CH} 11231$.

\section{Author contributions}

M.B. conceived the research. N.-E.L. derived the analytic expressions, implemented the code and carried out the numerical calculations. J.-J.Z. helped with code development. H.-Y.C. helped deriving the equations. N.-E.L. and M.B. wrote the manuscript. All authors contributed to analyzing the results and editing the manuscript.

\section{Competing interests}

The authors declare no competing interests.

\section{Additional information}

Supplementary information is available for this paper at https://doi.org/10.1038/s41467 020-15339-0.

Correspondence and requests for materials should be addressed to M.B.

Peer review information Nature Communications thanks the anonymous reviewer(s) for their contribution to the peer review of this work.

Reprints and permission information is available at http://www.nature.com/reprints

Publisher's note Springer Nature remains neutral with regard to jurisdictional claims in published maps and institutional affiliations.

(c) Open Access This article is licensed under a Creative Commons Attribution 4.0 International License, which permits use, sharing, adaptation, distribution and reproduction in any medium or format, as long as you give appropriate credit to the original author(s) and the source, provide a link to the Creative Commons license, and indicate if changes were made. The images or other third party material in this article are included in the article's Creative Commons license, unless indicated otherwise in a credit line to the material. If material is not included in the article's Creative Commons license and your intended use is not permitted by statutory regulation or exceeds the permitted use, you will need to obtain permission directly from the copyright holder. To view a copy of this license, visit http://creativecommons.org/ licenses/by/4.0/.

(c) The Author(s) 2020 